\title{
Article \\ Evaluation of a Passive Upper-Limb Exoskeleton Applied to Assist Farming Activities in Fruit Orchards
}

\author{
Hsien-Min Wang, Dang Khanh Linh Le and Wei-Chih Lin * \\ Department of Mechanical and Electro-Mechanical Engineering, National Sun Yat-sen University, \\ Kaohsiung 80424, Taiwan; d063020004@student.nsysu.edu.tw (H.-M.W.); \\ m083020102@student.nsysu.edu.tw (D.K.L.L.) \\ * Correspondence: wc.lin@mail.nsysu.edu.tw; Tel.: +886-7-5252000-4226
}

Citation: Wang, H.-M.; Le, D.K.L.; Lin, W.-C. Evaluation of a Passive Upper-Limb Exoskeleton Applied to Assist Farming Activities in Fruit Orchards. Appl. Sci. 2021, 11, 757. https://doi.org/10.3390/app11020757

Received: 11 December 2020 Accepted: 11 January 2021 Published: 14 January 2021

Publisher's Note: MDPI stays neutral with regard to jurisdictional clai$\mathrm{ms}$ in published maps and institutional affiliations.

Copyright: () 2021 by the authors. Licensee MDPI, Basel, Switzerland. This article is an open access article distributed under the terms and conditions of the Creative Commons Attribution (CC BY) license (https:// creativecommons.org/licenses/by/ $4.0 /)$.

\begin{abstract}
Work-related musculoskeletal disorder (WMSD) is a common occupational injury. Among the occupational injuries of agricultural workers, $93 \%$ are related to WMSD, with the shoulder being the second most affected area. This paper presents a passive upper-limb exoskeleton (PULE) with a gas spring and four-bar mechanism developed to aid the daily activities associated with orchard farming. The PULE is used to assist the arm-lifting process, reducing the physical exertion of farmers and the risk of developing WMSD. Electromyography (EMG) measurements of 26 participants were obtained to evaluate the difference in physical exertion with and without the PULE. Two arm activities: fruit thinning, with the arms raised and maintained at $100^{\circ}$, and pesticide spraying, with the hands swinging from $0^{\circ}$ to $100^{\circ}$ and back, were simulated. Using the PULE decreased muscle tension of the anterior deltoid (AD) by 17.64-19.86\%. The PULE also decreased the AD activity by $37.67-39.57 \%$ during the actual orchard farming operations. The Qualisys motion capture system indicated that the difference in the lifting angle of the upper limb with and without the PULE was less than $1^{\circ}$ and not significant $(\alpha>0.05)$. Thus, the PULE did not affect the flexibility of the wearer in orchard farming activities.
\end{abstract}

Keywords: work-related musculoskeletal disorders; upper-limb exoskeleton; four-bar mechanism; electromyography; muscle tension

\section{Introduction}

In 2015, the average age of individuals engaged in agricultural tasks in the Republic of China (ROC) was 57.1 yr. [1], which indicated that the ROC was suffering from an aging agrarian population. According to the statistical information of the ROC in 2013, orchard farmers aged between 25-44 yr. and 45-64 yr. accounted for $11.14 \%$ and $64.85 \%$ of all orchard farmers, respectively [2]. In recent years, the Council of Agriculture of the ROC has vigorously encouraged young people to engage in agricultural tasks, owing to which, the average age of the agricultural technology group in Kaohsiung has decreased to 41 yr. [3]. Nevertheless, orchard farming-related tasks, such as fruit thinning and pesticide spraying, require considerable muscle movement. During these arm-lifting movements, orchard farmers must repetitively lift their arms, leading to considerable physical exertion. Specifically, such repetitive motions of lifting the upper limbs generate significant body fatigue and reduce efficiency, especially in the case of elderly orchard farmers.

Work-related musculoskeletal disorders (WMSD) are painful conditions of the muscles, tendons, and neuralgia. WMSD is a common occupational injury caused by the overuse of a muscle without proper rest. In general, nearly all tasks require the use of arms and hands, and thus, WMSD of the upper limbs in the modern workplace must be addressed [4]. Furthermore, several ergonomic risk factors of WMSDs exist in agricultural tasks, particularly from overwork and non-neutral postures [5,6]. A report demonstrated that $93 \%$ of the occupational injuries of agricultural workers were related to WMSD. Carpal tunnel syndrome accounted for one-third of the WMSD cases, and the shoulder was the 
second most affected body part [7]. In recent years, automation and robot technology have been widely implemented, but the amount of workload has not significantly decreased. The load levels continue to be relatively high in the construction, agriculture, and manufacturing domains [8]. Even during low-intensity physical activities, muscle fatigue can occur, which can damage muscle function. The continuous contraction of muscles can restrict blood circulation and further increase muscle fatigue [9]. In particular, the repetitive lifting movements of the arms, which are required in orchard farming activities, can increase the risk of developing WMSD [10,11]. Therefore, research on the use of exoskeletons to help alleviate muscle fatigue has attracted much attention. In general, exoskeletons can be divided into active or passive systems. Active systems consist of one or more actuators to enhance the strength of the wearer. In contrast, passive systems employ gas springs or other materials as a power source that stores and releases energy to assist the wearer in performing physical activities $[12,13]$. With the development of various exoskeletons, the passive upper-limb exoskeleton (PULE) has emerged as a promising preventive measure to decrease upper-limb fatigue caused by repetitive lifting movements. In this regard, the PULE is composed of two parts, namely, the wearable passive power driving device and the support mechanism suit. The driving device enhances and assists human movement to reduce the physical exertion of the wearer, thereby reducing the wearer's risk of developing WSMD [12,14].

Existing research on PULEs is mostly focused on industrial tasks such as precision tasks and repeated manual material handling tasks in car factories. In these tasks, the PULE can effectively decrease the stress level of the wearer in lifting his/her arms. It has been demonstrated that, on average, the use of a PULE increased work performance by $30 \%$ and reduced the fatigue of the wearer [15]. In load lifting/lowering and box unstacking/stacking tasks, the use of a PULE reduced anterior deltoid (AD) muscle activity by $54 \%$ and $73 \%$, respectively [16]. In static overhead work with a $2 \mathrm{~kg}$ load, the PULE significantly decreased the biceps brachii muscle and medial deltoid activity by $49 \%$ and $62 \%$, respectively [17]. According to these results, using a PULE can help reduce muscle exertion during upper-limb activity; however, this aspect has been examined only in the industrial domain. In recent years, few upper-limb exoskeletons suitable for agricultural tasks have been studied. Some literature reviews mention the existence of passive backsupport exoskeletons applied to assist farming and industrial tasks [18-21], but these are not upper-limb exoskeletons. The PULEs presently available on the market are targeted at medical rehabilitation and industrial tasks, and they are not suitable for assisting the daily activities of orchard farmers. To this end, in this study, a PULE with a simple construction was developed and a patent (the application number of the patent in the ROC is 108116962) [22] was obtained. The proposed PULE can reduce the muscle activity required in the repetitive lifting of the upper limb necessary in orchard farming, thereby reducing the risk of developing WMSD. This PULE can assist orchard farmers in their daily activities and solve the problem related to the aging agricultural population in the ROC.

\section{Materials and Methods}

\subsection{Experimental Design}

The design of the experiment is divided into two parts: First, a simulation of the fruit thinning and pesticide spraying activities is conducted in the laboratory; Then, after verifying that the PULE can safely and effectively reduce muscle tension in arm-lifting activities, the PULE is worn to perform the actual operation of pesticide spraying on a pomelo tree and fruit thinning of a jujube tree.

- In the laboratory: Two common arm-lifting movements were simulated (fruit thinning and pesticide spraying) in the laboratory, and the participants were divided into groups A (25-40 yr.) and B (41-64 yr.) to compare the PULE effect in different age groups. Because this test was the first evaluation of the PULE, for safety reasons, the test movements were limited to simple short-term lifting exercises of the arms. The independent variables were the ages of groups A and B, two types of arm-related 
activities, namely, fruit thinning and pesticide spraying, and the use of the PULE (with PULE: ES, without PULE: W-ES). In this experimental design, the main muscle used to lift the upper limb and perform internal rotation was the AD muscle. Therefore, we measured electromyography (EMG) signals only for the $\mathrm{AD}$. The dependent variables were the EMG of the AD, the lifting angle of the upper limb $(\theta)$, and the ratings of perceived exertion (RPE) of the upper limb. Additionally, the local perceived pressure (LPP) was rated for the ES condition. The experiment considered four conditions (the lifting movements of the fruit thinning and pesticide spraying activities, W-ES, and ES), which were implemented by each participant. Two forms of arm activity (fruit thinning and pesticide spraying) were selected, as they are commonly performed during orchard farming.

- In the orchard: After confirming that the PULE can effectively and safely assist arm lifting in the laboratory, the farmers then performed the real operation of pesticide spraying for the pomelo tree and fruit thinning for the jujube tree. The independent variables were the use of the PULE (with PULE: ES, without PULE: W-ES). The dependent variables were the RPE of the upper limb and the EMG of the AD, lateral deltoid (LD), and posterior deltoid (PD). Additionally, the LPP was rated for the ES condition. In this experimental design, pesticide spraying and fruit thinning are continuous complicated actions that include internal rotation, abduction, and external rotation. The main muscles used to lift the upper limbs and undergo internal rotation, abduction, and external rotation were the $\mathrm{AD}, \mathrm{LD}$, and $\mathrm{PD}$. Therefore, we measured the EMG signals for the AD, $\mathrm{LD}$, and PD.

\subsection{Experimental Procedures}

- In the laboratory: The participants were briefed about the experimental procedures and equipment when they entered the laboratory. Subsequently, EMG electrodes were placed over the $\mathrm{AD}$ of the participants. Following a detailed demonstration and explanation by the researchers, the participants practiced the relevant movements until they became proficient. The participants were allowed to rest for five minutes between each step to avoid fatigue. The experiment involved the following steps:

Step 1: Simulation of fruit thinning without the PULE: Each participant raised their hand and held it at $100^{\circ}$ for $90 \mathrm{~s}$. The state in which the hand of the participant was naturally drooping was defined as $0^{\circ}$. This action was repeated three times with a five-minute break between each action to avoid fatigue.

Step 2: Simulation of pesticide spraying without the PULE: The participant was asked to move his hand from $0^{\circ}$ to $100^{\circ}$, swinging back and forth for 90 s., guided by a metronome with a frequency of $2 \mathrm{~s}$. This action was repeated three times with a five-minute break between each action to avoid fatigue.

Step 3: Steps 1 and 2 were repeated with the PULE. Figure 1a shows the image of the PULE on a participant during the fruit thinning simulation.

Step 4: The participants performed the maximum voluntary contractions (MVCs), and this action was repeated three times with a one-minute break between each action to avoid fatigue. To avoid fatigue of the AD before the experiment started, the MVC measurements were performed at the end.

- In the orchard: The pomelo farmers and jujube farmers were briefed about the experimental procedures and equipment when they entered the orchards. Subsequently, EMG electrodes were placed over the $\mathrm{AD}, \mathrm{LD}$, and $\mathrm{PD}$ of the farmers after a systematic demonstration and clarification by the researchers. The experiment in the orchard involved the following steps:

Step 1: The pesticide spraying of the pomelo tree or the fruit thinning of the jujube tree without the exoskeleton had a duration of $30 \mathrm{~min}$.

Step 2: The pesticide spraying of the pomelo tree or fruit thinning of the jujube tree with the exoskeleton had a duration of $30 \mathrm{~min}$. Figure $1 \mathrm{~b}$ shows the image of the pesticide 
spraying activity of the pomelo tree without the PULE. Step 1 and Step 2 were separated by more than twelve hours to avoid fatigue. Because the pomelo farmers must wear protective clothing during the entire pesticide spraying process, it was necessary to ensure that the EMG electrodes did not fall off during the experiment.

Step 3: Each farmer performed the MVCs, and this action was repeated three times with a one-minute break in between each action to avoid fatigue [23]. The MVC measurements were performed at the end to avoid fatigue of the AD, LD, and PD [17].

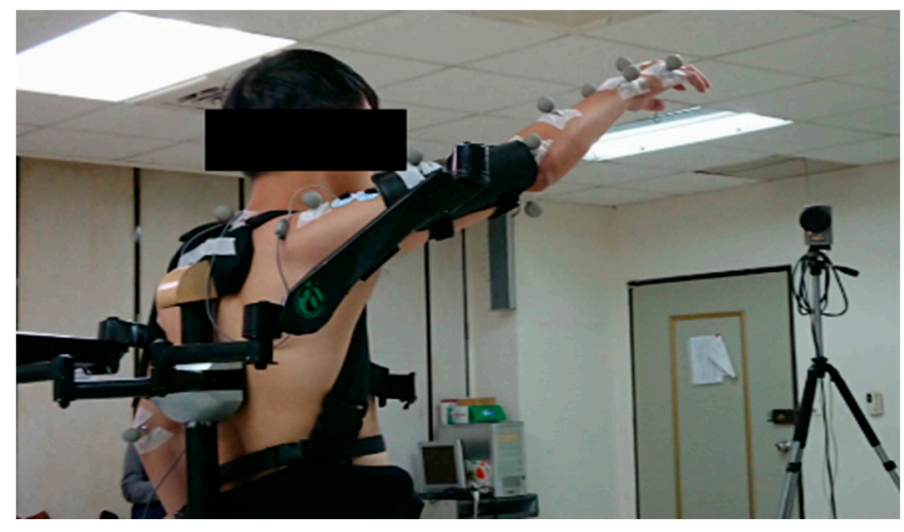

(a)

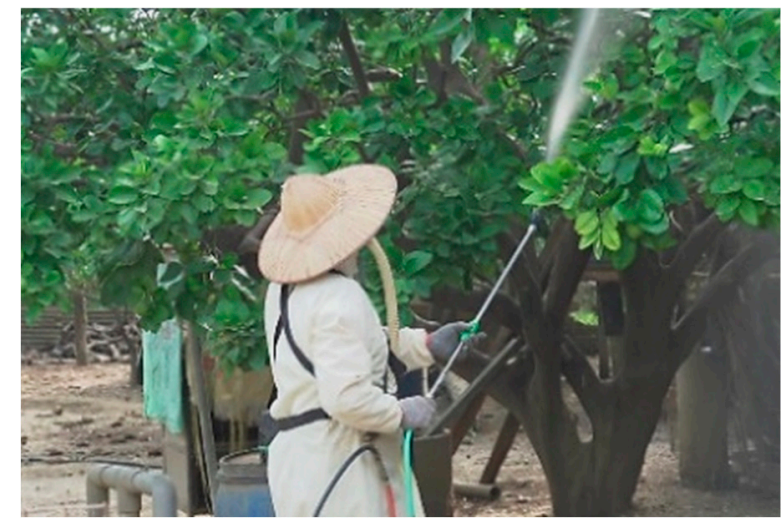

(b)

Figure 1. Experimental photographs. (a) Photograph of the passive upper-limb exoskeleton (PULE) during the simulation of fruit thinning; (b) Photograph of the pesticide spraying activity of the pomelo tree.

\subsection{Participants and Ethics Approval}

- In the laboratory: Similar to references [2,3], eighteen male participants aged 25-40 yr. and 41-64 yr. were divided into groups A and B, respectively. Group A consisted of 8 people, and group $B$ had 10 people. Consent was obtained from all the participants to participate in the experiment.

- In the orchard: Group C consisted of four male participants whose task was to spray pesticide on the pomelo tree. Group D consisted of three male participants and one female participant whose task was to perform fruit thinning for the jujube tree. To initiate the experiment, permission was obtained from all participants. The average and standard deviation (SD) for groups A, B, C, and D in terms of age, weight, and height are listed in Table 1. None of the participants had any musculoskeletal disorders. The Ethics Committee of Kaohsiung Veterans General Hospital approved the experimental design, and the IRB No. is KSVGH20-CT6-10.

Table 1. Mean parameters and SD for groups A $(n=8), \mathrm{B}(n=10), \mathrm{C}(n=4)$, and D $(n=4)$.

\begin{tabular}{cccc}
\hline Participants & Age (y) & Weight $\mathbf{( k g )}$ & Height $(\mathbf{m m})$ \\
\hline Group A & $30.3 \pm 5.38$ & $70.0 \pm 9.77$ & $1707.5 \pm 2.38$ \\
Group B & $50.3 \pm 9.02$ & $78.4 \pm 11.69$ & $1668.0 \pm 4.97$ \\
Group C & $50.5 \pm 6.38$ & $76.0 \pm 16.89$ & $1702.5 \pm 4.81$ \\
Group D & $53.0 \pm 11.97$ & $73.0 \pm 9.61$ & $1660.0 \pm 5.04$ \\
\hline
\end{tabular}

\subsection{Equipment}

\subsubsection{Exoskeleton}

The evaluated PULE was developed by the Biomimicking and Engineering Laboratory of the National Sun Yat-Sen University, Kaohsiung, Taiwan, the ROC [22]. This device is wearable with the objective of reducing muscle fatigue of the arms during the lifting process through a gas spring mounted on the robotic arm [24]. The PULE is composed of two parts: the wearable supporting-mechanism suit that weighs $3 \mathrm{~kg}$ and the passive 
power driving device with two arm-attachments that weigh $1.6 \mathrm{~kg}$ each, leading to a total PULE weight of $6.2 \mathrm{~kg}$. The user can wear the PULE like a backpack, and it can be adjusted and fixed to the body through the fixation belt located on the supporting-mechanism suit.

\subsubsection{Surface EMG}

The Noraxon Surface EMG System (TELEMYO 2400, Noraxon, Scottsdale, AZ, USA) was used to record the AD muscle activity changes of groups A and B as well as the AD, LD, and PD muscle activity changes of groups C and D. The EMG signal was at a sampling rate of $1000 \mathrm{~Hz}$, and the EMG electrodes were placed over the muscle measured for each participant with $20 \mathrm{~mm}$ between the electrodes. Before using the EMG electrodes, the skin was cleaned with an alcohol cleansing pad and shaved to reduce any noise. The EMG electrodes were fixed using elastic bands [23]. The grounding electrode was placed on the 7th cervical vertebra (C7), and the EMG information was examined and analyzed using MyoResearchXP Basic Edition 1.07.01 software. A bandpass filter at 80-250 Hz was added to the data using MyoResearch-XP 1.07 (Noraxon, Scottsdale, AZ, USA) software, and the EMG signals were rectified. The EMG signals used to measure muscle activities were processed using the root mean square (RMS) value to quantify the EMG signals. The EMG amplitude was calculated by the RMS values of the raw EMG. The EMG amplitude of each muscle was normalized by \%MVC. The \%MVC was compared with the RMS and MVC, as characterized by Equation (1). Equation (2) illustrates the PULE effect. The mean values of the amplitude were used to measure the degree of muscle contraction $[25,26]$, whereas the area under the amplitude curve was used to approximate muscle work [27].

$$
\begin{gathered}
\% M V C=\left(\frac{R M S_{\text {force application }}-R M S_{\text {force relaxation }}}{R M S_{M V C}-R M S_{\text {force relaxation }}}\right) * 100 \% \\
\text { the PULE effect }=\left(\frac{\% M V C_{W-E S}-\% M V C_{E S}}{\% M V C_{W-E S}}\right) * 100 \%
\end{gathered}
$$

\subsubsection{Qualisys Motion Capture System}

After the participants entered the laboratory, they were requested to stand on the force plate at the position marked by the researchers. The participants were required to stand in the same position during the experimental process. The researchers used a protractor to measure the position when a participant lifted his hand to $100^{\circ}$. A bracket was placed next to the participant to mark the position to assist the participants in promptly lifting their arm to that position. During the experiment, the researchers were always at the side of the participant to ensure that the lifting angle of the upper limb of the participant was constant.

The difference in the lifting angle of the upper limb during the simulation of the fruit thinning and pesticide spraying activities was recorded using a Qualisys motion capture system (Qualisys ${ }^{\mathrm{TM}}$, Gutenberg, Sweden), which was composed of three parts: six high-speed cameras, a force plate (Type 9286AA; Kistler, Switzerland) filtered at $10 \mathrm{~Hz}$ and sampled at $800 \mathrm{~Hz}$ with a precision of \pm 0.5 percent, and 24 sensors. During the $30 \mathrm{~s}$. of each experimental phase, the participants stood on the force plate while measurements were performed. During the experiment, the device tracked the trajectory of the hand of the participant. The sampling rate of the camera was $200 \mathrm{~Hz}$. Before beginning the experiment, 24 sensors were fastened onto the arms and trunk of the participant's body and infrared light was used to collect the sensor markings on the participant. The change in the trajectory of the sensors during the experiment was detected to simulate the movement of the body of the participants. Finally, the change in the lifting angle of the arms and trunk of the body was calculated using the Visual 3D (version 3.79.0) software tool.

\subsubsection{Perceived Exertion}

In terms of the participants' self-evaluation, the perceived exertion and postural discomfort were recorded using the Borg 6-20 RPE scale and LPP scale, respectively. The 
RPE scale ranges from six (very light) to twenty (maximum exertion) and represents a tool to evaluate the RPE based on self-observation [28]. The LPP scale, which ranges from zero (no pressure at all) to ten (extremely strong pressure), represents a tool to evaluate the perceived postural discomfort also based on self-observation $[29,30]$. The participants assessed the perceived pressure on the arms after wearing the PULE for each experimental step.

\subsubsection{The Torque Measurement}

Auxiliary forces are generated by the gas springs in the robotic arm of the PULE. To identify the relationship between the compressed angle of the robotic arm and the auxiliary force, we measured the robotic arm length $(R)$, the variation in the auxiliary forces $(F)$ on the glove of the robotic arm with a loadcell (10 kg, Straight Bar TAL220, SparkFun, Boulder, CO, USA), and the corresponding compressed angle ( $\theta$ ) of the robotic arm with an MPU6050 sensor (GY-521 MPU6050 6DOF, InvenSense, San Jose, CA, USA) based on Arduino-UNO (ITALY). The initial compressed angle of the robotic arm of the PULE was $71^{\circ}$, which was set as $0^{\circ}$, and the compression test was initiated to obtain the corresponding equation and coefficient of determination (R-squared) through $\mathrm{R}, \mathrm{F}$, and $\theta$. The R-squared parameter is a measure of how close certain data are to the equation, and for correspondence, the $\mathrm{R}$-squared should be higher or equal to $95 \%$ [31]. Figure 2 shows a photograph of the loadcell and MPU6050 sensor.

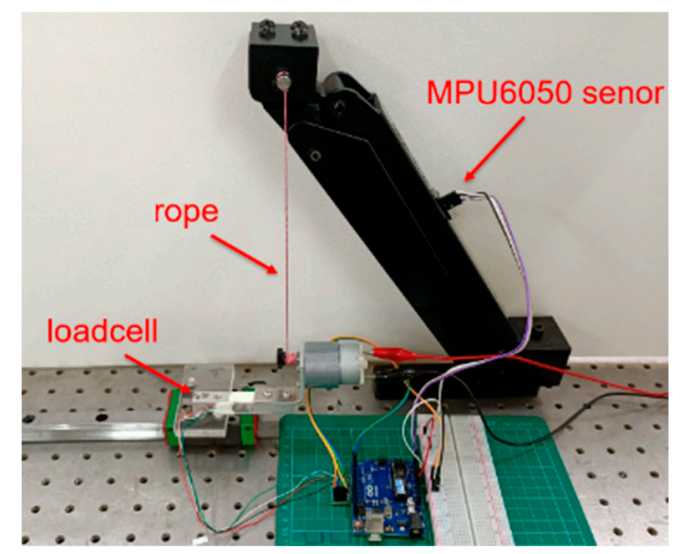

Figure 2. Photograph of the loadcell and MPU6050 sensor.

\subsubsection{Data Analysis}

One-way analysis of variance (ANOVA) was utilized to analyze all the information from the laboratory experiments, and the significance was set as $\alpha<0.05$. The RPE scale of the upper limb before and after wearing the PULE, the \%MVC of the AD before and after wearing the PULE, and the difference in the lifting angle of the upper limb before and after wearing the PULE for fruit thinning and pesticide spraying were analyzed. Each dependent variable of the participants was analyzed, and ten analyses were performed, as listed in Table 2. 
Table 2. One-way ANOVA was performed for each of the conditions being analyzed (A: group A, B: group B, W-ES: Without PULE, ES: With PULE).

\begin{tabular}{ccc}
\hline Analysis & Independent Variable & Dependent Variable \\
\hline 1 & A_W-ES_thinning vs. A_ES_thinning & \%Maximum voluntary \\
2 & A_W-ES_ spraying vs. A_ES_ spraying & contractions (\%MVC) \\
3 & B_W-ES_thinning vs. B_ES_thinning & \\
4 & B_W-ES_ spraying vs. B_ES_spraying & \\
\hline 5 & A_W-ES_thinning vs. A_ES_thinning & Ratings of perceived exertion \\
6 & A_W-ES_ spraying vs. A_ES_ spraying & (RPE) \\
7 & B_W-ES_thinning vs. B_ES_thinning & \\
8 & B_W-ES_ spraying vs. B_ES_ spraying & Lifting angle of the upper \\
9 & W-ES_thinning vs. ES_thinning & limb $(\theta)$ \\
\hline 10 & W-ES_ spraying vs. ES_spraying &
\end{tabular}

\section{Results}

Due to a malfunctioning of the surface EMG system, only 6 and 9 data points during the laboratory experiment for groups A and B, respectively, could be analyzed. There was contact between the EMG electrodes and the PULE during the testing process, resulting in EMG signals that exhibited a certain interference [30].

\subsection{Muscle Activity}

In the fruit thinning simulation, the PULE reduced the \%MVC of the AD from 28.6 to $22.92(\alpha=0.407)$ and from 28.57 to $23.53(\alpha=0.176)$ for groups $A$ and $B$, respectively. Similarly, in the pesticide spraying simulation, the \%MVC of the AD decreased from 39.53 to $31.78\left(\alpha=2.212 \times 10^{-11}\right)$ and from 39.08 to $31.54(\alpha=0.055)$ for groups A and B, respectively. These results indicate that the PULE induced reduction in the AD muscle activity. The \%MVC of the AD for groups A and B with and without using the PULE for the fruit thinning and pesticide spraying activities is shown in Figure 3. Figure 4 shows that the PULE reduced the \%MVC of the AD from 55.74 to 33.68 and from 21.66 to 13.5 for groups $C$ and $D$, respectively.

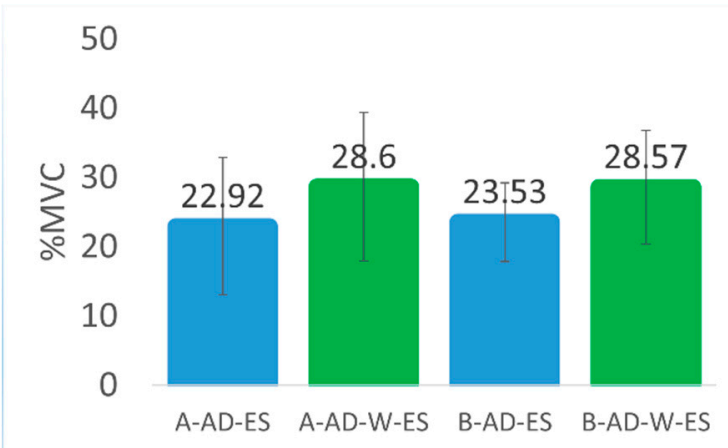

(a)

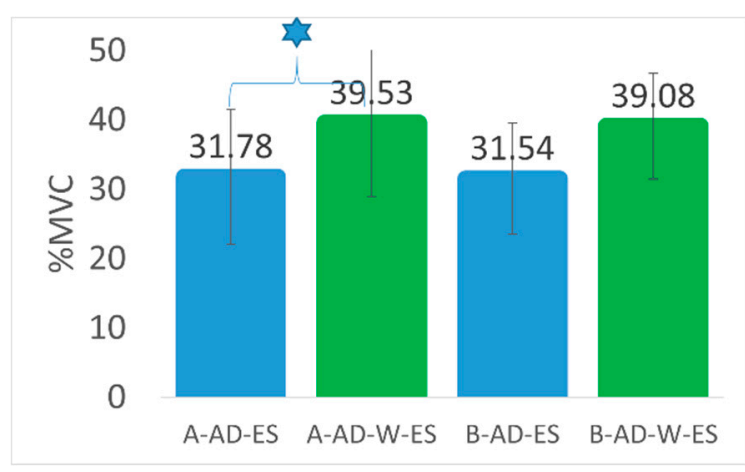

(b)

Figure 3. The \%MVC of the anterior deltoid (AD) for groups A and B with and without using the PULE in the: (a) fruit thinning simulation; (b) pesticide spraying simulation. The star symbol indicates statistical significance $(\alpha<0.05)$. 


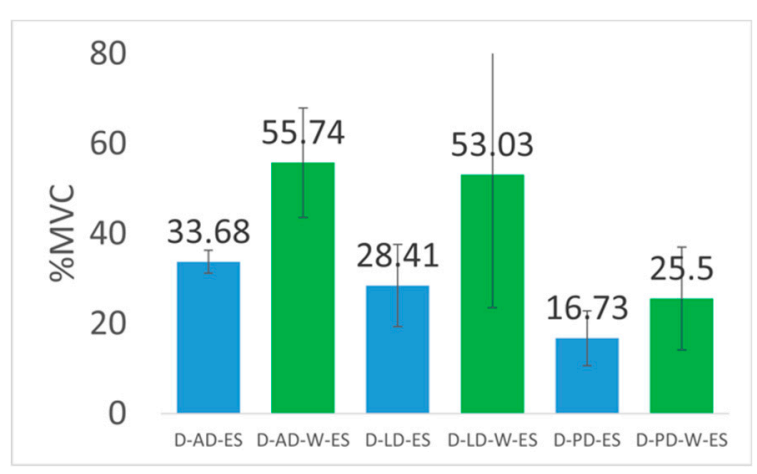

(a)

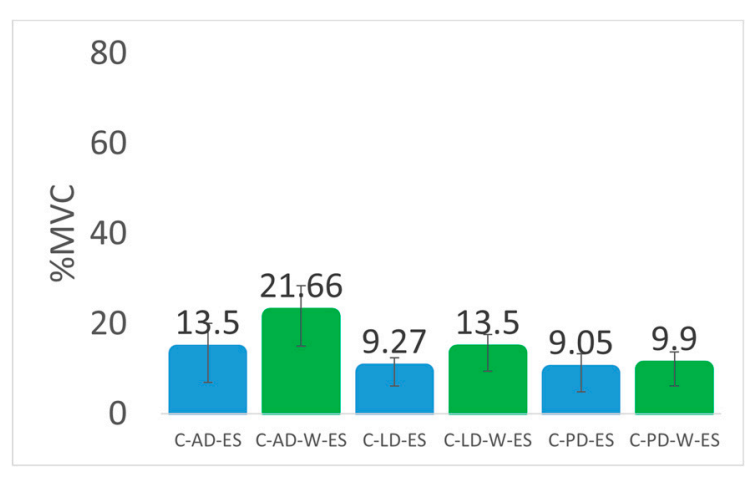

(b)

Figure 4. The \%MVC of the AD, lateral deltoid (LD), and posterior deltoid (PD) for groups C and D with and without using the PULE for: (a) fruit thinning of the jujube tree; (b) pesticide spraying on the pomelo tree.

\subsection{Kinematics}

In the laboratory, the change in the lifting angle of the upper limb before and after wearing the PULE for fruit thinning and pesticide spraying was observed using the Qualisys motion capture system. According to the experimental time, the change in the lifting angle of the 18 participants involved three stages, namely, the early stage $(0-10 \mathrm{~s})$, middle stage (45-55 s), and last stage (80-90 s). The results indicate that the mean change in the lifting angle in the three stages for fruit thinning and pesticide spraying was less than $1^{\circ}$ and not statistically significant $(\alpha>0.05)$. Tables 3 and 4 list the mean change in the lifting angle of the upper limb with the SD and the $\alpha$ value for the cases with and without using the PULE for the fruit thinning and pesticide spraying activities, respectively.

Table 3. Mean change in the lifting angle of the upper limb and $\alpha$ value before and after wearing the PULE for fruit thinning simulation $(n=18)$.

\begin{tabular}{cccc}
\hline Time & PULE & Mean \pm SD & $\alpha$ Value \\
\hline \multirow{2}{*}{$0-10(\mathrm{~s})$} & ES & $100.88 \pm 4.38$ & 0.844 \\
& W-ES & $101.23 \pm 3.99$ & 0.899 \\
\hline \multirow{2}{*}{$45-55(\mathrm{~s})$} & ES & $99.73 \pm 4.71$ & \multirow{2}{*}{0.881} \\
& W-ES & $99.98 \pm 4.29$ & \\
\hline \multirow{2}{*}{$80-90(\mathrm{~s})$} & ES & $98.95 \pm 4.87$ & \\
& W-ES & $98.87 \pm 4.38$ & \\
\hline
\end{tabular}

Table 4. Mean change in the lifting angle of the upper limb and $\alpha$ value before and after wearing the PULE for pesticide spraying simulation $(n=18)$.

\begin{tabular}{cccc}
\hline Time & PULE & Mean \pm SD & $\alpha$ Value \\
\hline \multirow{2}{*}{$0-10(\mathrm{~s})$} & ES & $101.68 \pm 6.90$ & 0.825 \\
& W-ES & $101.77 \pm 4.48$ & 0.6 \\
\hline \multirow{2}{*}{$45-55(\mathrm{~s})$} & ES & $100.68 \pm 7.01$ & \multirow{2}{*}{0.588} \\
& W-ES & $100.4 \pm 4.08$ & \\
\hline \multirow{2}{*}{$80-90(\mathrm{~s})$} & ES & $100.19 \pm 6.77$ & \\
& W-ES & $99.73 \pm 4.48$ & \\
\hline
\end{tabular}

\subsection{Borg 6-20 RPE Scale}

In the fruit thinning simulation, the PULE reduced the Borg 6-20 RPE scale values from 11.62 to $8.87\left(\alpha=4.908 \times 10^{-5}\right)$ and from 11.7 to $9.3\left(\alpha=2.292 \times 10^{-5}\right)$ for groups A and $\mathrm{B}$, respectively. Similarly, in the pesticide spraying simulation, the RPE scale values 
decreased from 8.87 to $7.37\left(\alpha=1.473 \mathrm{E} \times 10^{-4}\right)$ and from 8.9 to $7.3\left(\alpha=1.939 \times 10^{-5}\right)$ for groups A and B, respectively. The PULE-induced reduction in the mean RPE scale of the upper limb was statistically significant $(\alpha<0.05)$. The mean RPE scale of the upper limb for groups A and B with and without using the PULE for fruit thinning and pesticide spraying is shown in Figure 5. Figure 6 shows that the PULE reduced the mean RPE scale from 8 to 6.75 and from 11.25 to 10.25 for groups $C$ and $D$, respectively.

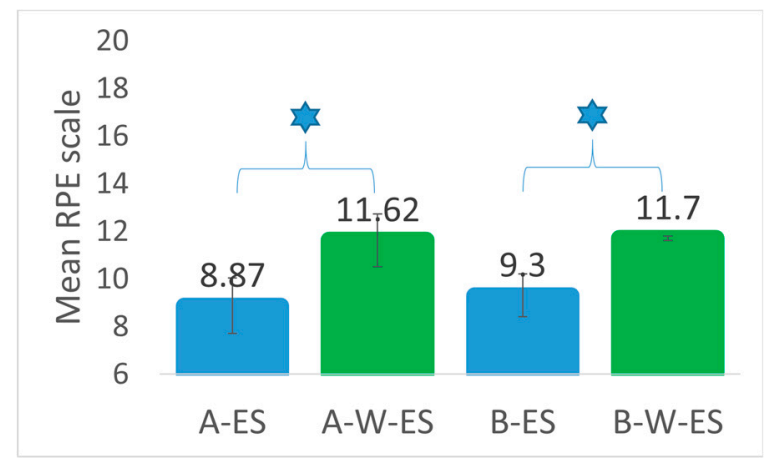

(a)

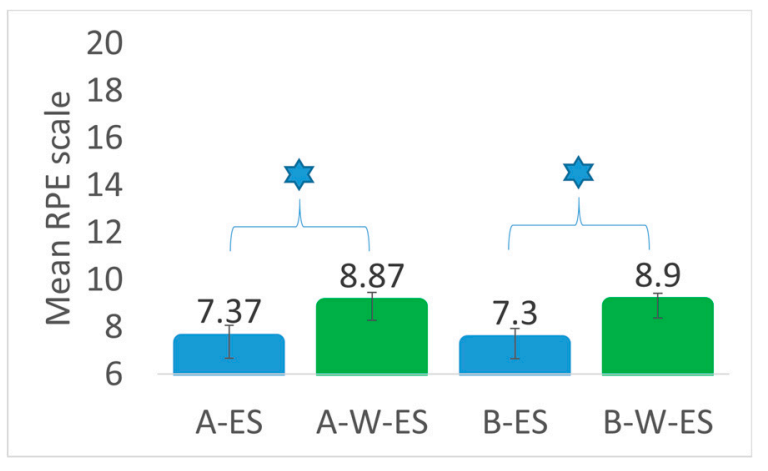

(b)

Figure 5. Mean RPE scale of the upper limb for groups A and B with and without using the PULE for: (a) fruit thinning simulation; (b) pesticide spraying simulation. The star symbol indicates statistical significance $(\alpha<0.05)$.

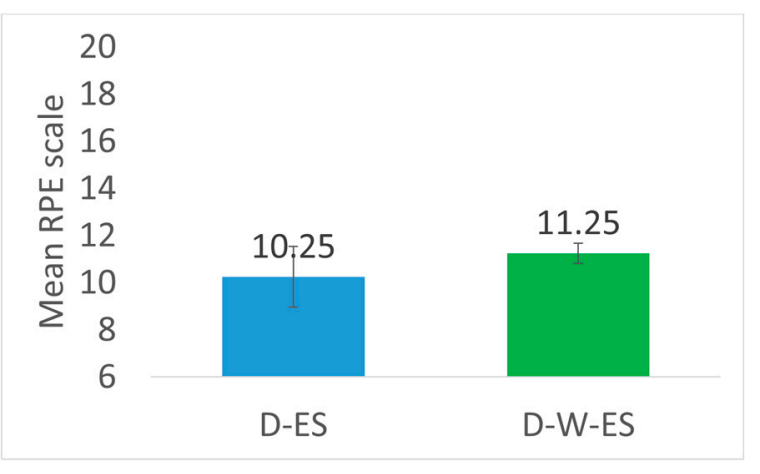

(a)

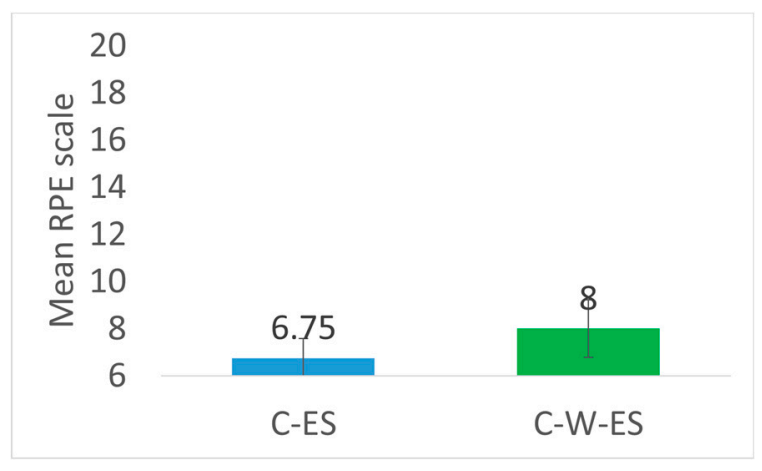

(b)

Figure 6. Mean RPE scale of the upper limb for groups C and D with and without using the PULE for: (a) fruit thinning of the jujube tree; (b) pesticide spraying of the pomelo tree.

\subsection{LPP Scale}

In the fruit thinning simulation, the LPP scale value for the upper limb with the PULE was 1.0 and 1.1 for groups A and B, respectively. Similarly, in the pesticide spraying simulation, the LPP scale value was 0.5 and 0.6 for groups A and B, respectively. The mean LPP scale value was 1.75 and 0.25 for groups $C$ and $D$, respectively. The mean LPP scale values of the upper limb using the PULE is shown in Figure 7. 


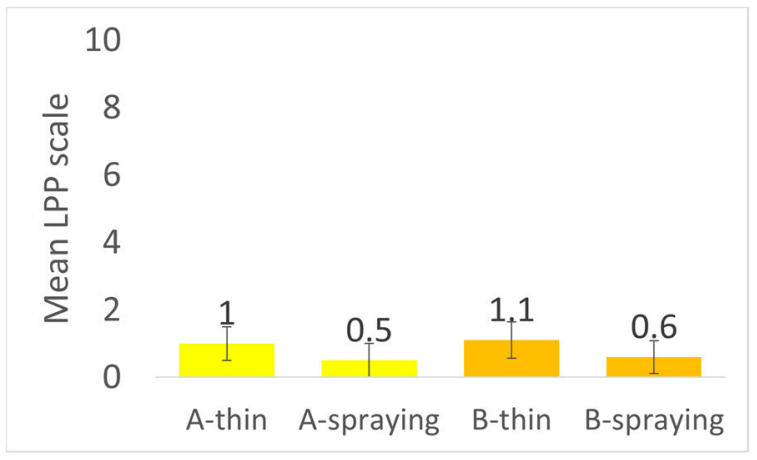

(a)

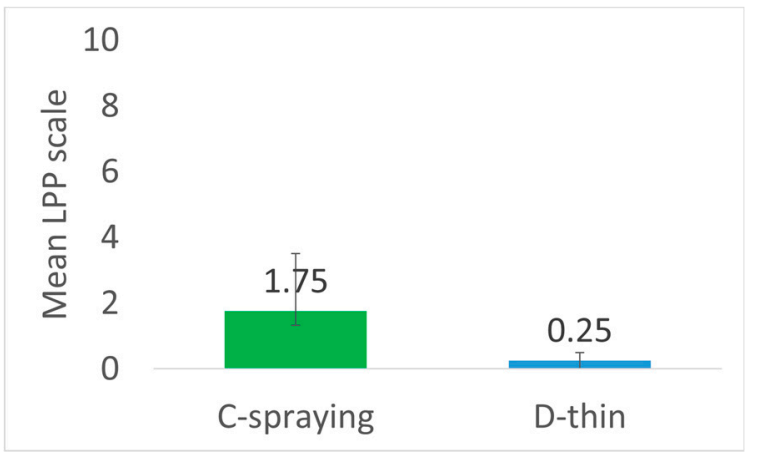

(b)

Figure 7. Mean LPP scale of the upper limb using the PULE for: (a) fruit thinning and pesticide spraying simulations for groups $\mathrm{A}$ and $\mathrm{B} ;(\mathbf{b})$ fruit thinning of the jujube tree and pesticide spraying of the pomelo tree for groups $\mathrm{C}$ and $\mathrm{D}$.

\subsection{The Torque Measurement}

Figure 8 shows the relationship between the torque (M) and the corresponding compressed angle of the robotic arm. The angle change $(\theta)$ and corresponding auxiliary force (F) of the robotic arm were measured from $0^{\circ}$ to $61^{\circ}$ using the MPU6050 and loadcell sensor. The results indicate that the parameters exhibited a linear regression relation. Equation (3) shows the relationship between the torque of the robotic arm and $\theta$. The R-squared value was $96.56 \%$.

$$
M=0.2214 \theta-0.665
$$

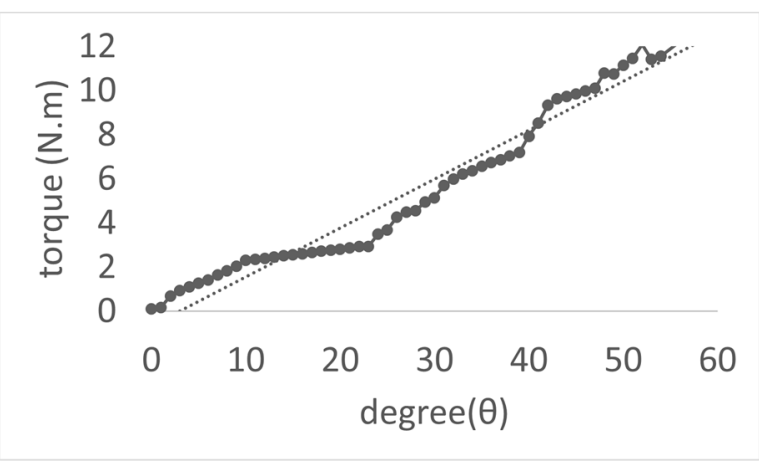

Figure 8. Linear regression for the torque of the PULE.

\section{Discussion}

This study evaluated the ergonomics of the PULE to provide auxiliary forces for the upper limb during orchard farming.

\subsection{Muscle Activity}

The purpose of the PULE is to reduce the upper limb muscle tension of the person wearing the PULE. With a decrease in muscle tension, fatigue is expected to be lessened, reducing the risk of developing WMSD in the upper limb [17]. The PULE demonstrated a decrease in the AD muscle activity of $17.64 \%$ and $19.86 \%$ for groups $\mathrm{A}$ and $\mathrm{B}$, respectively, for which the experiments were conducted in the laboratory. Similarly, the PULE demonstrated a decrease in the AD muscle activity of $37.67 \%$ and $39.57 \%$ for groups $\mathrm{C}$ and $\mathrm{D}$, respectively, for which the experiments were conducted in the orchard. This decrease in the AD muscle activity indicates that the PULE can effectively reduce the muscle tension experienced by orchard farmers. The PULE effect observed in the orchard farming simulation was significantly lower than that of the actual orchard farming operation. This 
phenomenon is likely due to the fact that the actual operation actions include internal rotation, abduction, and external rotation, for which the PULE could also decrease the LD and PD muscle activities.

The \%MVC of the AD for groups A and B during pesticide spraying was higher than that during fruit thinning, indicating that pesticide spraying requires a larger muscle activity of the upper limb compared to the muscle tension required for fruit thinning. However, the RPE scale of groups A and B for pesticide spraying was lower than that for fruit thinning, indicating that the participants felt that pesticide spraying was easier than fruit thinning. This phenomenon is likely due to the short-term lowering of the upper limb during pesticide spraying. Specifically, the participants moved their hand from $0^{\circ}$ to $100^{\circ}$, swinging back and forth, which provided the AD with a brief rest, and consequently, the participants felt less tired.

The PULE reduced the \%MVC of the AD by 22.06 for the jujube thinning activity. These results are superior to those of the upper limb assist device developed by the Institute of Agricultural Machinery of Japan, which reduced the \%MVC of grape thinning by 8 [32]. The proposed PULE also decreased the LD muscle activity for the jujube thinning activity by $46.42 \%$. These results are worse than the $62 \%$ decrease in the static overhead work with a 2-kg load [17]. This phenomenon is likely due to the long-term raising of the upper limb during the jujube thinning activity, as static overhead work with a 2-kg load requires raising the upper limb for only $30 \mathrm{~s}$.

In groups A and B, the PULE reduced the work required by the AD muscle by $17.64 \%$ to $19.86 \%$, but there is no significant difference. The reason for this is likely because the torque of the PULE is constant and cannot be adjusted. If the torque can be modified, the upper limb lifting action may be able to benefit from a greater auxiliary gain for the participants.

Moreover, although a 20-yr. age difference existed between groups A and B, the PULE effect was similar for both fruit thinning and pesticide spraying activities. In other words, the PULE effect did not exhibit any significant difference between groups A and B in both the activities. This result indicates that the age of the participants (25-64 yr.) was not a factor in the PULE effect.

\subsection{Kinematics and Perceived Exertion}

In the laboratory, the mean improvement in the upper limb raising angle was less than $1^{\circ}$ for the fruit thinning and pesticide spraying activities. These findings show that the PULE did not impact the flexibility of the wearers in the orchard farming activities.

The RPE scale values measured with the PULE in all groups were lower than those without the PULE, indicating that the PULE was able to reduce the perceived exertion as determined by the self-evaluation of the participants. The LPP scale values for all groups were less than 2, equivalent to a "weak pressure" [33]. Overall, this is a direct effect, as the comfortability of wearing the exoskeleton is one of the main factors affecting the acceptability of exoskeleton use [12]. The LPP scale value for groups A and B during fruit thinning was 1.0 and 1.1, respectively, and the results were similar to those for a static overhead task with the exoskeleton involving no load [17].

\subsection{The Torque Measurement}

The torque of the designed exoskeleton EKSOVest is generated by a high-pressure spring. The relationship between the torque and corresponding compressed angle of the EKSOVest was simulated to be from $1^{\circ}\left(2.08\right.$ N.m) to $81^{\circ}(9.12$ N.m) [31]. In this study, the torque of the PULE is generated by a gas spring on the robotic arm, and the torque and angle of the PULE were measured to be from $0^{\circ}\left(0.09\right.$ N.m) to $61^{\circ}(12.76$ N.m). These values indicate that the PULE provides more torque than the EKSOVest.

The R-squared value of the linear regression relation was $96.56 \%$. This value confirmed the reliability of the obtained regression, as it could well simulate the relationship between the torque and angle of the PULE. The action of the gas spring was determined with 
Equation (3) and corresponded to the linear regression. These results show that the PULE can provide a maximum auxiliary force of $43.878 \mathrm{~N}$ at a $61^{\circ}$ angle of the PULE. According to the characteristics of the human limbs, a single upper limb of a male accounts for $5.74 \%$ of the total body weight [34]. In other words, for a male weighing $78 \mathrm{~kg}$, the single upper limb corresponds to a force of approximately $43.876 \mathrm{~N}$. Thus, the PULE is most suitable for wearers weighing $78 \mathrm{~kg}$.

\subsection{Limitations}

Because most orchard farmers are elderly in the ROC, the current overall weight of the PULE $(6.2 \mathrm{~kg}$ ) may be too heavy for most farmers. Therefore, an attempt must be made to reduce the weight of the PULE in future work.

The torque of the PULE is constant and cannot be adjusted in terms of the strength of the individual upper limb muscle. If the torque can be adjusted, certain individuals may be able to benefit from more auxiliary aid for arm-lifting activities. Therefore, future work may also include rendering the torque of the PULE adjustable in future variants.

\section{Conclusions}

The PULE, developed for orchard farming applications, is composed of a wearable supporting-mechanism suit, which can be fixed to the body through the fixation belt, and a passive power driving device that provides auxiliary force to the arm during the lifting activity of the upper limb. In this study, the PULE demonstrated a decrease in the AD muscle activity ranging from $17.64 \%$ to $19.86 \%$ during the simulation of fruit thinning and pesticide spraying activities. The PULE also demonstrated a decrease in the AD activity ranging from $37.67 \%$ to $39.57 \%$ during the actual operation of orchard farming. The Qualisys motion capture system indicated that the PULE did not affect the flexibility of the wearer in orchard farming activities. Finally, the results of the RPE scale also indicate that the PULE reduces the perceived exertion as determined by the participants' self-evaluation. The measurement results confirmed that the proposed PULE can assist in arm-lifting motions to decrease the physical exertion of orchard farmers, thereby reducing the risk of developing WMSD in the upper limbs.

\section{Patents}

In this study, the proposed and evaluated PULE was developed by the Biomimicking and Engineering Laboratory of the National Sun Yat-sen University, Kaohsiung, Taiwan, and has obtained a patent (the application number of the patent is 108116962) [22].

Author Contributions: Conceptualization, H.-M.W. and W.-C.L.; methodology, H.-M.W. and W.-C.L.; software, H.-M.W. and D.K.L.L.; writing—original draft preparation, H.-M.W.; writing-review and editing, H.-M.W. and W.-C.L.; project administration, W.-C.L. All authors have read and agreed to the published version of the manuscript.

Funding: This research was supported by the Council of Agriculture, ROC (project numbers 108AS13.2.11-ST-a1, 109AS-11.3.1-ST-a1 and 110AS-8.3.1-ST-a1).

Institutional Review Board Statement: The study was conducted according to the guidelines of the Declaration of Helsinki, and approved by the Ethics Committee of Kaohsiung Veterans General Hospital (protocol code KSVGH20-CT6-10 and approval date: 27 May 2020).

Informed Consent Statement: Informed consent was obtained from all subjects involved in the study.

Acknowledgments: The authors gratefully acknowledge the assistance, facilities, and support supplied by the NSYSU-Biomimicking and Engineering Lab (Being2 Lab) National Sun Yat-sen University, Kaohsiung, Taiwan.

Conflicts of Interest: The authors declare no conflict of interest. 


\section{References}

1. Survey Results of Income of Main Farmers in 2015. Available online: https:/ / www.coa.gov.tw/ws.php?id=2505701 (accessed on 10 September 2020).

2. The Most Important Workers in Farming and Animal Husbandry Are Classified by Gender and Age. Available online: https: / / www.stat.gov.tw / ct.asp? xItem=37583\&CtNode=5017\&mp=4 (accessed on 9 September 2020).

3. Announcement Information of Kaohsiung District Agricultural Research and Extension Station. Available online: https://www. kdais.gov.tw / theme_data.php?theme=news\&sub_theme=announcement\&id=7525 (accessed on 11 September 2020).

4. Shin, S.-J.; Yoo, W.-G.; Kim, T.-Y. Effects of different overhead work conditions on the neck and shoulder muscles. J. Phys. Ther. 2012, 24, 197-199. [CrossRef]

5. Davis, K.G.; Kotowski, S.E. Understanding the ergonomic risk for musculoskeletal disorders in the United States agricultural sector. Am. J. Ind. Med. 2007, 50, 501-511. [CrossRef] [PubMed]

6. Meyers, J.M.; A Miles, J.; Faucett, J.; Janowitz, I.; Tejeda, D.G.; Weber, E.; Smith, R.; Garcia, L. Priority Risk Factors for Back Injury in Agricultural Field Work. J. Agromed. 2002, 8, 39-54. [CrossRef]

7. Bernard, C.; Tourne, M. Musculoskeletal disorders in agriculture. La Revue du Praticien 2007, 57, 45-50. [PubMed]

8. Parent-Thirion, A.; Biletta, I.; Cabrita, J.; Vargas, O.; Vermeylen, G.; Wilczynska, A.; Wilkens, M. Book Eurofound: Sixth European Working Conditions Survey-Overview Report (2017 Update); Office of the European Union: Luxembourg, 2017.

9. Ng, D.; McNee, C.; Kieser, J.; Farella, M. Neck and shoulder muscle activity during standardized work-related postural tasks. Appl. Ergon. 2014, 45, 556-563. [CrossRef] [PubMed]

10. da Costa, B.R.; Vieira, E.R. Risk factors for work-related musculoskeletal disorders: A systematic review of recent longitudinal studies. Am. J. Ind. Med. 2009, 53, 285-323. [CrossRef]

11. Jaffar, N.; Abdul-Tharim, A.H.; Mohd-Kamar, I.F.; Lop, N.S. A literature review of ergonomics risk factors in construction industry. In Proceedings of the 2nd International Building Control Conference, Penang, Malaysia, 11-12 July $2011 ;$ pp. 89-97.

12. De Looze, M.P.; Bosch, T.; Krause, F.; Stadler, K.S.; O'Sullivan, L.W. Exoskeletons for industrial application and their potential effects on physical work load. Ergonomics 2016, 59, 671-681. [CrossRef]

13. Matthew, R.P.; Mica, E.J.; Meinhold, W.; Loeza, J.A.; Tomizuka, M.; Bajcsy, R. Introduction and initial exploration of an active/passive exoskeleton framework for portable assistance. In Proceedings of the 2015 IEEE/RSJ International Conference on Intelligent Robots and Systems (IROS), Hamburg, Germany, 28 September-2 October 2015; IEEE: Piscataway, NJ, USA, 2015; pp. 5351-5356.

14. Romero, D.; Stahre, J.; Wuest, T.; Noran, O.; Bernus, P.; Fast-Berglund, Å.; Gorecky, D. Towards an operator 4.0 typology: A human-centric perspective on the fourth industrial revolution technologies. In Proceedings of the international Conference on Computers and industrial Engineering (CIE46), Tianjin, China, 29-31 October 2016; pp. 29-31.

15. Spada, S.; Ghibaudo, L.; Gilotta, S.; Gastaldi, L.; Cavatorta, M.P. Investigation into the Applicability of a Passive Upper-limb Exoskeleton in Automotive Industry. In Proceedings of the 27th International Conference on Flexible Automation and Intelligent Manufacturing, Modena, Italy, 27-30 June 2017; pp. 1255-1262.

16. Theurel, J.; Desbrosses, K.; Roux, T.; Savescu, A. Physiological consequences of using an upper limb exoskeleton during manual handling tasks. Appl. Ergon. 2018, 67, 211-217. [CrossRef]

17. Huysamen, K.; Bosch, T.; Looze Md Stadler, K.S.; Graf, E.; O'Sullivan, L.W. Evaluation of a passive exoskeleton for static upper limb activities. Appl. Ergon. 2018, 70, 148-155. [CrossRef]

18. Omoniyi, A.; Trask, C.; Milosavljevic, S.; Thamsuwan, O. Farmers' perceptions of exoskeleton use on farms: Finding the right tool for the work (er). Int. J. Ind. Ergon. 2020, 80, 103036. [CrossRef]

19. Wei, W.; Wang, W.; Qu, Z.; Gu, J.; Lin, X.; Yue, C. The effects of a passive exoskeleton on muscle activity and metabolic cost of energy. Adv. Robot. 2020, 34, 19-27. [CrossRef]

20. Ippolito, D.; Constantinescu, C.; Rusu, C.A. Enhancement of human-centered workplace design and optimization with Exoskeleton technology. Procedia CIRP 2020, 91, 243-248. [CrossRef]

21. Thamsuwan, O.; Milosavljevic, S.; Srinivasan, D.; Trask, C. Potential exoskeleton uses for reducing low back muscular activity during farm tasks. Am. J. Ind. Med. 2020, 63, 1017-1028. [CrossRef]

22. Patent Announcement of People's Republic of China. Available online: https://twpat3.tipo.gov.tw/tipotwoc/tipotwkm?@@1754 12089 (accessed on 15 September 2020).

23. Hermens, H.J.; Freriks, B.; Disselhorst-Klug, C.; Rau, G. Development of recommendations for SEMG sensors and sensor placement procedures. J. Electromyogr. Kinesiol. 2000, 10, 361-374. [CrossRef]

24. Altenburger, R.; Scherly, D.; Stadler, K.S. Design of a passive, iso-elastic upper limb exoskeleton for gravity compensation. Robomech. J. 2016, 3, 12. [CrossRef]

25. De Luca, C.J. The use of surface electromyography in biomechanics. J. Appl. Biomech. 1997, 13, 135-163. [CrossRef]

26. Rau, G.; Schulte, E.; Disselhorst-Klug, C. From cell to movement: To what answers does EMG really contribute? J. Electromyogr. Kinesiol. 2004, 14, 611-617. [CrossRef] [PubMed]

27. Gallo, L.; Salis Gross, S.; Palla, S. Nocturnal masseter EMG activity of healthy subjects in a natural environment. J. Dent. Res. 1999, 78, 1436-1444. [CrossRef] [PubMed]

28. Borg, G. Borg's Perceived Exertion and Pain Scales; Human Kinetics: Champaign, IL, USA, 1998. 
29. Van der Grinten, M.P.; Smitt, P. Development of a practical method for measuring body part discomfort. Adv. Ind. Ergon. Saf. 1992, 4, 311-318.

30. Huysamen, K.; de Looze, M.; Bosch, T.; Ortiz, J.; Toxiri, S.; O'Sullivan, L.W. Assessment of an active industrial exoskeleton to aid dynamic lifting and lowering manual handling tasks. Appl. Ergon. 2018, 68, 125-131. [CrossRef]

31. Hoyos Rodriguez, D. Realistic Computer Aided Design: Model of an Exoskeleton. Master's Thesis, University of Skövde, School of Engineering Science, Skövde, Sweden, 2019.

32. A Brief Introduction to the Development and Current Situation of Japanese Fruit Cultivation Machinery. Available online: https: / / www.coa.gov.tw / ws.php?id=2508271 (accessed on 11 September 2020).

33. Hamberg-van Reenen, H.H.; Van Der Beek, A.J.; Blatter, B.M.; Van Der Grinten, M.P.; Van Mechelen, W.; Bongers, P.M. Does musculoskeletal discomfort at work predict future musculoskeletal pain? J. Ergon. 2008, 51, 637-648. [CrossRef] [PubMed]

34. The Characteristics of Human Limbs in Taiwan. Available online: https://www.ilosh.gov.tw/menu/1188/1201/ (accessed on 9 September 2020). 